\title{
Finite Temperature Magnetism in Gd: Evidence against a Stoner Behavior
}

\author{
K. Maiti,* M. C. Malagoli, A. Dallmeyer, and C. Carbone ${ }^{\dagger}$ \\ Institut für Festkörperforschung, Forschungszentrum Jülich, D-52428 Jülich, Germany
}

(Received 19 September 2001; published 5 April 2002)

\begin{abstract}
The temperature dependence of the rare-earth valence bands has been regarded as a realization of the Stoner behavior. The exchange splitting of the electronic states appears to scale as the magnetic order parameter for $T<T_{C}$ and to vanish at $T=T_{C}$. We report here a spin-resolved photoemission study on the evolution of Gd bulk bands for $0.5 \leq T / T_{C} \leq 1$. The spin-polarized spectral line shapes display a complex temperature dependence, which clearly contrasts with the interpretation of previous experimental results. The spin-resolved photoemission data demonstrate the inadequacy of the Stoner model to the description of magnetism in rare earths.
\end{abstract}

DOI: $10.1103 /$ PhysRevLett.88.167205

Understanding the relation between electronic and magnetic properties at finite temperatures is a central question for many branches of solid state physics. While the electronic theory accurately accounts for the ground state properties of magnetic materials, it meets only limited success in describing their finite temperature behavior, such as the magnetic phase transitions, the corresponding ordering temperatures, and critical parameters.

Two simple models are usually considered to schematically describe the finite temperature behavior of the electronic structure, depending on the degree of the electron localization. The Stoner model [1] predicts that the exchange splitting of delocalized states parallels the temperature dependence of the magnetization. In this case, the exchange-split electronic subbands gradually merge together and become degenerate at the Curie temperature, $T_{C}$. The spin-mixing model [2], on the other hand, describes strongly localized systems where thermal fluctuations of the local magnetic moment directions reduce the magnetization, but leave the magnitude of the local moments and the local electronic structure unchanged. Thus, the exchange splitting of the electronic states does not vary according to this model, while the characteristic spin polarization of the states is gradually lost on approaching $T_{C}$.

Several experimental studies have examined the finite temperature magnetism in the elemental ferromagnets. The itinerant $3 d$-transition metals ( $\mathrm{Fe}, \mathrm{Co}$, and $\mathrm{Ni}$ ) show an intermediate behavior. The complexity of the temperature dependent effects on the electronic structure in these systems defies any simple description [3-5]. Rare earths, on the other hand, provide a different and apparently simpler scenario. The magnetic moment in these systems primarily originates from the unfilled $4 f$ subshell, which displays a simple spin-mixing behavior due to its atomiclike character. The magnetic coupling between the $4 f$ moments occurs via the highly delocalized $(5 d 6 s)$-valence states [6]. Various investigations by spin-integrated photoemission spectroscopy (PES) and inverse photoemission spectroscopy (IPES) report that the exchange splitting of the bulk valence bands vanishes at $T_{C}[7-11]$. These experimental results have been interpreted as a first evidence
PACS numbers: 75.70.Ak, 75.25.+z, 79.60.Bm

for the applicability of the Stoner model to real ferromagnetic systems.

Gadolinium (Gd) is the most investigated case, being a prototype Heisenberg ferromagnet with a large moment $(S=7 / 2)$ in the half-filled $4 f$ levels. The exchange interaction with the $4 f$ states leads to a large exchange splitting $(1 \mathrm{eV})$ of the delocalized $(5 d 6 s)$-valence bands. In accord to the Stoner model, the exchange splitting of the bulk valence states, $\Delta_{\mathrm{ex}}^{\text {bulk }}$, is observed to decrease with increasing temperatures in PES [7,8] and IPES [9] measurements, and to vanish at the bulk Curie temperature $\left(T_{\mathrm{Cb}}=293 \mathrm{~K}\right)$. It is interesting to notice that a higher degree of localization for the $5 d_{z^{2}}$-surface states of $\operatorname{Gd}(0001)$ surface leads to controversial results. A scanning tunneling spectroscopy study reports the persistence of the surface state exchange splitting, $\Delta_{\mathrm{ex}}^{\text {surface }}$, at and above $T_{\mathrm{Cb}}$ [12]. PES and IPES studies on the surface states reached strongly contrasting conclusions, ranging from pure spin mixing to Stoner behavior $[9,13,14]$.

While most attention has been devoted to the finite temperature behavior of the localized surface states, the bulk bands have been until now indisputably believed to follow a Stoner behavior. In the present study, we investigate the temperature dependence of Gd bulk and surface states by means of spin-resolved photoemission spectroscopy. The spin-resolved spectra provide evidence for a clear inadequacy of the Stoner model in describing the bulk band behavior. The failure of the model is manifested by a complex temperature dependence of the spin-resolved spectral line shapes, which cannot be observed in spin-integrated measurements. These results establish that changes in both the spin polarization and the exchange splitting of valence band states play a significant role on the magnetism of rare-earth metals.

Spin- and angle-resolved PES measurements were performed at the TGM5 wiggler-undulator beam line at BESSY I, Berlin. Experiments were carried out at normal emission with $34 \mathrm{eV}$ photon energy, in order to minimize the spectral broadening effect due to the photoelectron lifetime [6]. The spin polarization was measured by means of a Mott detector operating at 
$100 \mathrm{keV}$ and resolution $160 \mathrm{meV}$. Thirty monolayer (ML) thick $\operatorname{Gd}(0001)$ films were epitaxially grown by e-beam evaporation on a clean $\mathrm{W}(110)$ surface at room temperature $\left(P<2 \times 10^{-10} \mathrm{mbar}\right)$ and subsequently annealed at $600 \mathrm{~K}$. The cleanliness and crystalline structure of the samples were confirmed by the negligible intensities of impurity-related features in PES and by sharp LEED patterns, respectively.

We show spin-integrated and spin-resolved spectra of Gd measured at various temperatures in Fig. 1(a) and 1(b), respectively. The intense feature close to $E_{F}$ is due to $5 d_{z^{2-}}$ surface states and has a predominantly up-spin character. With increasing temperature, the spin polarization of these states continuously decreases and vanishes close to room temperature [see Fig. 1(b)]. Correspondingly, the peak position slightly shifts towards $E_{F}$, and the emission intensity decreases [Fig. 1(a)]. A simulation of the spin-integrated surface feature by a Lorentzian convoluted with the Fermi function and energy resolution (Gaussian) (solid lines in the figure) suggests that the peak position remains below $E_{F}$ up to room temperature, consistent with previous observations $[12,14]$. The phonon broadening is estimated to introduce a small increase $(\sim 0.1 \mathrm{eV})$ in the linewidth of the surface feature.
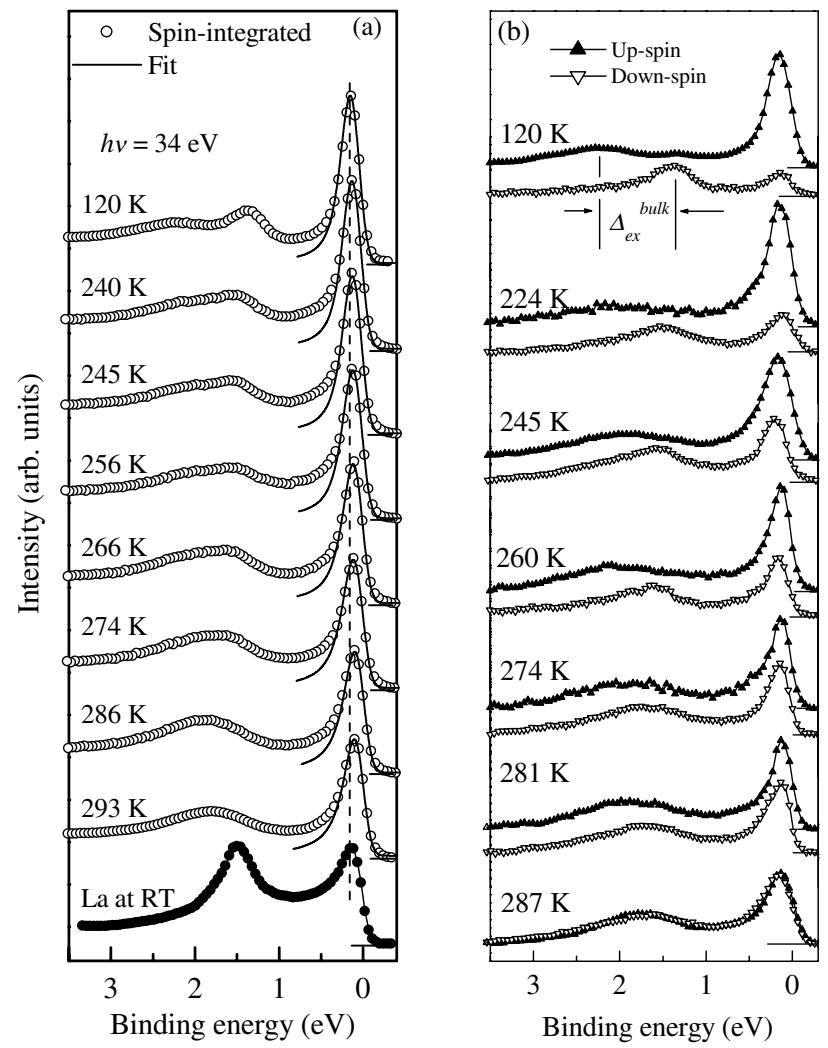

FIG. 1. (a) Spin-integrated and (b) spin-polarized spectra at different temperatures. The solid lines are the fit to the surface peak. Solid circles in (a) correspond to La reported from Ref. [16].
Relatively broader structures at higher binding energies are derived from $(5 d 6 s)$-bulk states having $\Delta_{2}$ symmetry. The spectra at $120 \mathrm{~K}$ exhibit up- and down-spin features around 2.3 and $1.4 \mathrm{eV}$ suggesting $\Delta_{\mathrm{ex}}^{\text {bulk }} \approx 0.9 \mathrm{eV}$, a value close to that estimated theoretically $(\sim 1 \mathrm{eV}$ at $T=0 \mathrm{~K})$ $[8,15]$. Interestingly, the distinct signature of the up- and down-spin states in Fig. 1(a) gradually disappears with the increase in temperature, suggesting a reduction of $\Delta_{\mathrm{ex}}^{\mathrm{bulk}}$. This is the key observation which led in previous studies to the conclusions of a Stoner behavior of the Gd $\Delta_{2}$ bulk bands. We note, however, that the linewidth of the $\mathrm{Gd}$ $\Delta_{2}$ band at $293 \mathrm{~K}$ is more than 2 times larger than that of nonmagnetic La [16]. Since the crystal structure and the valence configuration $\left[(5 d 6 s)^{3}\right]$ are very similar for $\mathrm{La}$ and $\mathrm{Gd}$, the above comparison suggests that the larger linewidth of the Gd $\Delta_{2}$ band might have a magnetic origin.

It is, thus, important to examine the spin character of the spectral functions, in order to better understand the temperature dependence of the electronic structure. The spinresolved data in Fig. 1(b) indicate that $\Delta_{\text {ex }}^{\text {bulk }}$ decreases with temperature. In addition, they display a clear modification of the line shape as a function of temperature. We redraw the spin-resolved bulk features in Fig. 2(a) at an enhanced scale for better clarity. It can easily be recognized that the line shape of each spin component becomes much broader at intermediate temperatures, a fact which cannot be visualized in the spin-integrated measurements. These spectral changes are not expected in the Stoner picture. The binding energy, $E$ dependence, of the linewidth (FWHM) of the up- (up triangles) and down-spin (down triangles) features is shown in Fig. 2(b). It is evident that, as the center of gravity of the down-spin emission shifts to higher binding energies, its FWHM first increases and then decreases. A similar trend is also found for the up-spin features. The contribution to the linewidth due to the photohole lifetime is expected to increase continuously with $E$. However, the FWHM shown in Fig. 2(b) contrasts with this expectation and can obviously not be explained by phonon broadening.

A closer examination of the spin-resolved spectral line shape indicates that the spectral asymmetry changes significantly with temperature. A single-peak structure in each spin channel cannot account for the evolution of the spectral line shapes. Two components are most evident at $120 \mathrm{~K}$, where their energy separation is significantly larger than their linewidth. At higher temperatures, the spinresolved curves exhibit a significant spectral intensity in correspondence of the peak position in the opposite spin channel. This spectral weight appears to grow with the increasing temperature, effectively reducing the spin polarization of the features.

We simulate the bulk spectral functions in terms of two Voigt functions (combination of Lorentzian and Gaussian) after a linear background subtraction. The Gaussian and Lorentzian components account for the resolution and lifetime broadening, respectively. Relatively small effects 

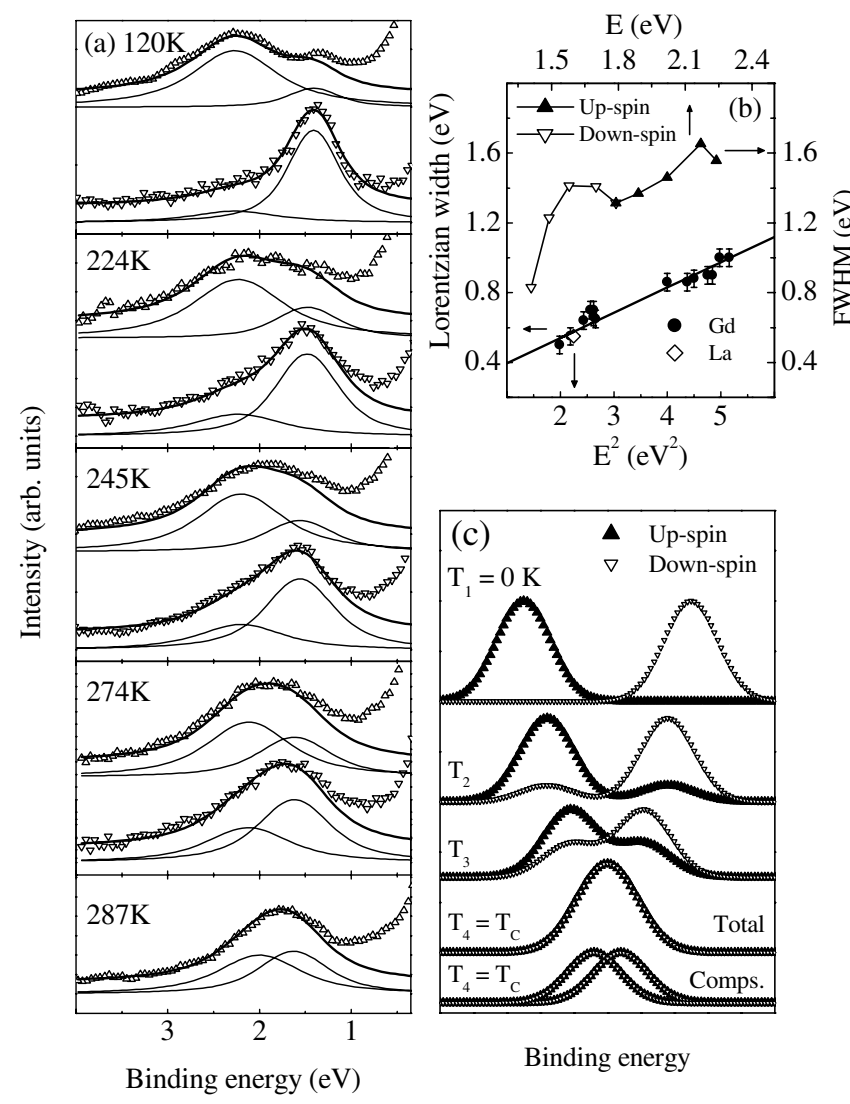

Binding energy

FIG. 2. (a) Spin-resolved spectra of $(5 d 6 s)$-bulk emissions at different temperatures. The up and down triangles represent the spectra corresponding to up- and down-spin states. The thick solid line is the simulated total spectra and the thin lines are the components. (b) Triangles represent the FWHM of the experimentally observed up- and down-spin features as a function of $E$. Simulated Lorentzian widths of the components are shown by circles as a function of $E^{2}$. The solid line shows the linear dependence of the Lorentzian widths. (c) Schematic model describing the finite temperature magnetism.

due to phonon broadening $(\sim 0.1 \mathrm{eV})$ are neglected here. The best fit is obtained via a least squared error method. The simulated spectra are shown by thick solid lines in Fig. 2(a). Interestingly, all the spectra could be described consistently only with two Voigt functions. The relative integrated intensity and the spectral width of the two components in the down-spin spectra are exactly reproduced (with reversed intensity ratio) in the up-spin ones, providing confidence to our simulations. Thus, besides a reduction of the exchange splitting, the spin polarization of the individual $\Delta_{2}$-emission features decreases with increasing temperature.

Since the dispersion close to the $\Gamma$ point is flat and the angular resolution is $<0.5^{\circ}$, the Lorentzian widths obtained here essentially reflect the photohole lifetime effects. We plot the Lorentzian widths (FWHM) as a function of $E^{2}$ in Fig. 2(b). The results may be described by a linear dependence ( $\gamma=\gamma_{0}+\alpha \times E^{2} ; \gamma=$ FWHM), suggesting a Fermi liquid behavior of the system as observed in other systems [17]. The values of $\gamma_{0}$ and $\alpha$ are $0.25 \mathrm{eV}$ and $0.15 \mathrm{eV}^{-1}$, respectively. It is to note here that the photohole lifetime may exhibit spin dependence due to unequal occupation of the spin-polarized bands above the photohole energy. However, the data in Fig. 2(b) do not show a clear evidence of such an effect. All the data points are within the error bars on a line. Interestingly, the open diamond representing the linewidth of the bulk feature of $\mathrm{La}$ [16] agrees well with the simulated data. The consistency of the above analysis with the experimental results suggests attributing the large linewidth of the Gd bulk feature at room temperature mainly to the persistence of a nonnegligible exchange splitting at $T \cong T_{\mathrm{Cb}}$.

The qualitative scenario is shown schematically in Fig. 2(c). At $T_{1}=0 \mathrm{~K}$, the up- and down-spin bands are separated by exchange splitting with $100 \%$ spin polarization. At finite temperatures, the exchange splitting reduces, and, as in the spin-mixing model, also the spin polarization decreases, as shown successively for $T=T_{2}$ and $T_{3}\left(T_{2}<\right.$ $T_{3}$ ). Thus, the spectra in each spin channel will show two components with relative intensities depending on the temperature. Finally, the spin polarization vanishes at the Curie temperature $\left(T_{4}=T_{C}\right)$. In this case, the spectral shape has to be identical in both spin channels and corresponds to the spin-integrated spectrum. The presence of an exchange splitting at this temperature or above cannot be detected in the spectra, unless it is comparable with the intrinsic linewidth of the individual peaks. In fact, the lifetime broadening for the bulk feature is too large $(\geq 0.7 \mathrm{eV})$ to observe a distinct signature of the exchange splitting, if this is smaller than $\approx 0.5 \mathrm{eV}$ at $T_{\mathrm{Cb}}$.

We now compare the exchange splitting and the spin polarization for surface and bulk states in Figs. 3(a) and 3 (b), respectively. The values of $\Delta_{\mathrm{ex}}^{\text {surface }}$ are reproduced from Bode et al. [12]. According to the results of the above analysis, the exchange splitting decreases with the increase in temperature and remains finite above $T_{\mathrm{Cb}}$, both at the surface and in the bulk. In addition, the spin polarization reduces continuously and approaches zero for $T=T_{\mathrm{Cb}}$. This indicates that the magnetization vanishes near $T_{\mathrm{Cb}}$ for both surface and bulk [18]. Spin polarization measurements [19] for out-of-plane magnetization at $T \geq T_{\mathrm{Cb}}$ rule out the possibility of canted magnetic moments at these temperatures. A more rapid decrease of the surface spin polarization compared to the bulk is consistent with the observations on other ferromagnetic systems [3-5], and also with a recent investigation on Gd [18].

The present results, thus, provide a direct evidence that the valence band in bulk Gd cannot be described within a purely Stoner or spin-mixing model. Theoretical calculations based on the combination of many-body interactions and ab initio band structure by Rex et al. [20] and Nolting et al. [21] predict a temperature behavior strongly dependent on the degree of itinerancy of the corresponding electronic states and on the specific $k$ point in the Brillouin zone. It is shown that the behavior of the $(5 d 6 s)$-valence 

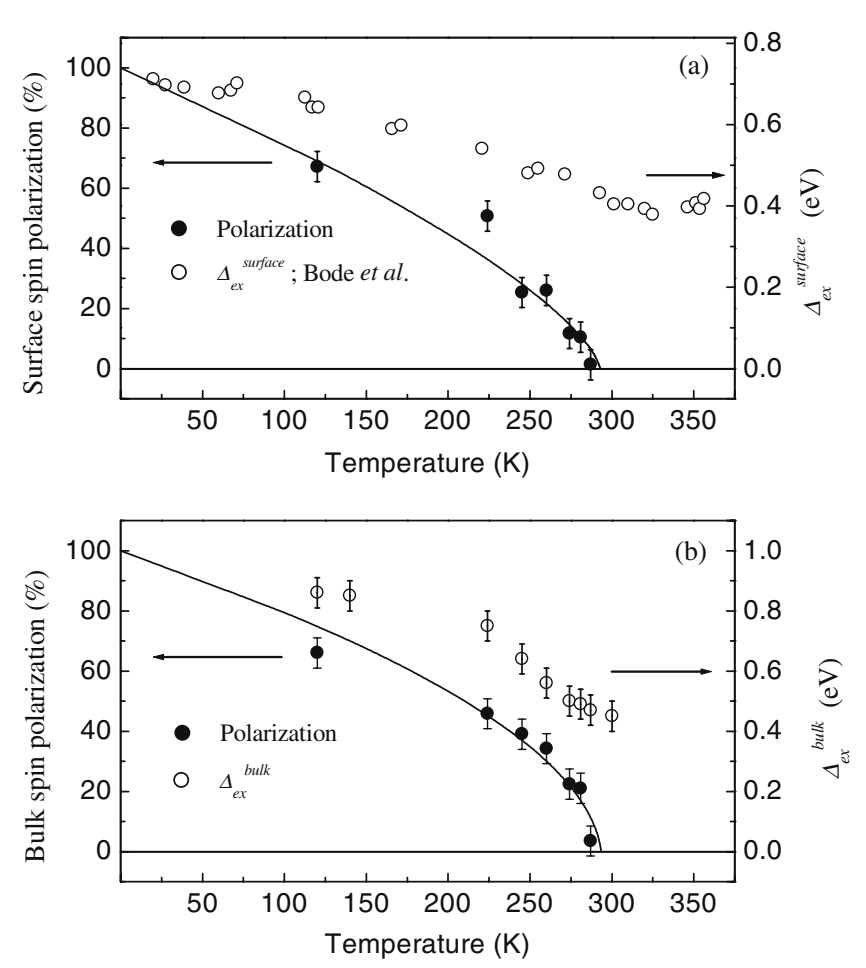

FIG. 3. (a) Temperature dependence of the spin polarization (solid circles) and exchange splitting (open circles) for the (a) surface and (b) bulk states. The bulk polarizations are obtained from the fitted results. The solid lines show a guide to the eye. The exchange splitting for the surface states has been reproduced from Ref. [12].

states can be strongly influenced by the degree and the type of local ordering of the $4 f$ moments, assumed in modeling the macroscopically disordered magnetic state. The overall theoretical trend shows that weakly correlated $s$-like states exhibit a temperature dependence closer to the Stoner picture, while relatively more localized $d$-like states approach a spin-mixing behavior. The general behavior is, however, intermediate between these two extreme cases, as reported in the present study. The bearing of these findings is likely significant for the other rare-earth metals as well, due to the very similar character of their band states. It is interesting to note here that other magnetic rare earths also exhibit a large linewidth of the $\Delta_{2}$ band near $T_{C}$, with only a small difference above and below the transition temperatures $[10,11,16]$.

In summary, we have investigated the temperature dependence of the electronic structure in Gd by means of spin-resolved photoemission spectroscopy. The spinresolved spectra reveal a complex temperature dependence, that could not be detected in spin-integrated measurements. In contrast to previous interpretations, the present results firmly establish that the Stoner picture cannot describe the Gd bulk bands.

K. M. thanks Alexander von Humboldt Stiftung and DLR, the Federal Republic of Germany, for financial assistance.

*Present address: Department of Condensed Matter Physics and Materials Science, Tata Institute of Fundamental Research, Homi Bhabha Road, Colaba, Mumbai-400005, India.

${ }^{\dagger}$ Present address: Consiglio Nazionale delle Ricerche, Istituto di Struttura della Materia, Sez. Trieste, Science Area Park, S.S. 163.5, Basovizza-Trieste, Italy.

[1] E. C. Stoner, Proc. R. Soc. London A 154, 656 (1936).

[2] V. Korenman et al., Phys. Rev. B 16, 4032 (1977); 16, 4048 (1977); H. Capellman, Z. Phys. B 34, 29 (1979); A. J. Pindor et al., J. Phys. F 13, 979 (1983); H. Hasegawa, J. Phys. Soc. Jpn. 46, 1504 (1979).

[3] E. Kisker, K. Schröder, M. Campagna, and W. Gudat, Phys. Rev. Lett. 52, 2285 (1984); J. Kirschner, M. Globl, V. Dose, and H. Scheidt, Phys. Rev. Lett. 53, 612 (1984).

[4] C. M. Schneider et al., Europhys. Lett. 16, 689 (1991).

[5] E. M. Haines, V. Heine, and A. Ziegler, J. Phys. F 16, 1343 (1986); K.-P. Kämper, W. Schmitt, and G. Güntherodt, Phys. Rev. B 42, 10696 (1990).

[6] K. Maiti, M. C. Malagoli, E. Magnano, A. Dallmeyer, and C. Carbone, Phys. Rev. Lett. 86, 2846 (2001).

[7] D. Li, J. Zhang, P. A. Dowben, and M. Onellion, Phys. Rev. B 45, 7272 (1992).

[8] B. Kim et al., Phys. Rev. Lett. 68, 1931 (1992).

[9] M. Donath, B. Gubanka, and F. Passek, Phys. Rev. Lett. 77, 5138 (1996).

[10] M. Bode et al., Phys. Rev. Lett. 83, 3017 (1999).

[11] C. Schüßler-Langeheine et al., Phys. Rev. Lett. 84, 5624 (2000).

[12] M. Bode et al., Appl. Phys. A 66, S121 (1998).

[13] D. Li et al., Phys. Rev. B 51, 13895 (1995).

[14] E. Weschke et al., Phys. Rev. Lett. 77, 3415 (1996).

[15] R. Wu, C. Li, A. J. Freeman, and C. L. Fu, Phys. Rev. B 44, 9400 (1991).

[16] E. Weschke and G. Kaindl, J. Electron. Spectrosc. Relat. Phenom. 75, 233 (1995).

[17] T. Valla, A. V. Fedorov, P. D. Johnson, and S. L. Hulbert, Phys. Rev. Lett. 83, 2085 (1999).

[18] C. S. Arnold and D. P. Pappas, Phys. Rev. Lett. 85, 5202 (2000).

[19] A. V. Federov et al., J. Electron. Spectrosc. Relat. Phenom. 92, 19 (1998).

[20] S. Rex, V. Eyert, and W. Nolting, J. Magn. Magn. Mater. 192, 529 (1999).

[21] W. Nolting, T. Dambeck, and G. Borstel, Z. Phys. B 94, 409 (1994). 